\title{
Conditional IL-4/IL-13-deficient mice reveal a critical role of innate immune cells for protective immunity against gastrointestinal helminths
}

\author{
K Oeser ${ }^{1}$, C Schwartz ${ }^{1}$ and D Voehringer ${ }^{1}$
}

Approximately one-third of the world population is infected with gastrointestinal helminths. Studies in mouse models have demonstrated that the cytokines interleukin (IL)-4 and IL-13 are essential for worm expulsion, but the critical cellular source of these cytokines is poorly defined. Here, we compared the immune response to Nippostrongylus brasiliensis in wild-type, Tcell-specific IL-4/IL-13-deficient and general IL-4/IL-13-deficient mice. We show that Tcell-derived IL-4/IL13 promoted T helper 2 (Th2) polarization in a paracrine manner, differentiation of alternatively activated macrophages, and tissue recruitment of innate effector cells. However, innate IL-4/IL-13 played the critical role for induction of goblet cell hyperplasia and secretion of effector molecules like Mucin5ac and RELM $\beta$ in the small intestine. Surprisingly, Tcellspecific IL-4/IL-13-deficient and wild-type mice cleared the parasite with comparable efficiency, whereas IL-4/IL-13deficient mice showed impaired expulsion. These findings demonstrate that IL-4/IL-13 produced by cells of the innate immune system is required and sufficient to initiate effective type 2 immune responses resulting in protective immunity against $N$. brasiliensis.

\section{INTRODUCTION}

Gastrointestinal helminth infections are a global health problem especially in countries with poor hygiene standards. Although drug treatments are available, reinfections are very common and can cause morbidity and mental retardation in children. ${ }^{1}$ That is why the development of effective vaccines should be a major focus of today's medical research, and in order to achieve this goal, it is essential to fully understand the host immune responses against helminths. Infections of mice with the gastrointestinal helminth Nippostrongylus brasiliensis constitute a well-established model for studying hookworm infections. The third-stage larvae of this parasite infect mice through the skin, migrate into the lung, are coughed up, and swallowed. Then they develop into mature adults that reside in the gut lumen where they produce eggs that are expelled in the feces. In wild-type (WT) mice the parasites themselves are expelled within 2 weeks. ${ }^{2}$ The infection causes a strong type 2 immune response with high immunoglobulin $\mathrm{E}$ (IgE) levels and increased numbers of T helper 2 (Th2) cells, eosinophils, basophils, and innate type 2 lymphoid cells (ILC2s).
Concerning the differentiation process of Th2 cells, the transcription factor Gata3 has been shown to be necessary and sufficient for the development of potent Th2 cells. ${ }^{3}$

The cytokines interleukin (IL)-4 and IL-13 are essential for orchestration of worm expulsion from the small intestine. IL-4 binds to the type I IL-4 receptor (IL-4R) that is mainly expressed on hematopoietic cells and consists of the common cytokine-receptor gamma subunit and the IL- $4 \mathrm{R} \alpha$ chain. ${ }^{4}$ However, both IL-4 and IL-13 are able to signal through the type II IL- 4 receptor complex that consists of the IL- $4 \mathrm{R} \alpha$ chain and the IL-13R $\alpha$ I chain. ${ }^{5}$ After infection with $N$. brasiliensis, mice with a complete deletion of the IL- $4 \mathrm{R} \alpha$ chain show an impaired differentiation of Th2 cells and a higher worm burden, ${ }^{6}$ whereas $\mathrm{T}$ cell-specific deletion of IL-4R $\alpha$ results in worm expulsion comparable to WT mice accompanied by normal goblet cell hyperplasia and high IgE levels. ${ }^{7} \mathrm{CD}^{+}{ }^{+} \mathrm{T}$ cells are clearly required for worm expulsion, ${ }^{8}$ and it was assumed that T cell-derived IL-4/IL-13 is critical for elimination of this parasite. However, we have previously shown that transfer of IL-4/IL-13-deficient T cells into $\mathrm{Rag}^{-/-}$mice is

${ }^{1}$ Department of Infection Biology, Institute of Microbiology, Universitätsklinikum Erlangen and Friedrich-Alexander-University Erlangen-Nürnberg (FAU), Erlangen, Germany. Correspondence: D Voehringer (david.voehringer@uk-erlangen.de) 

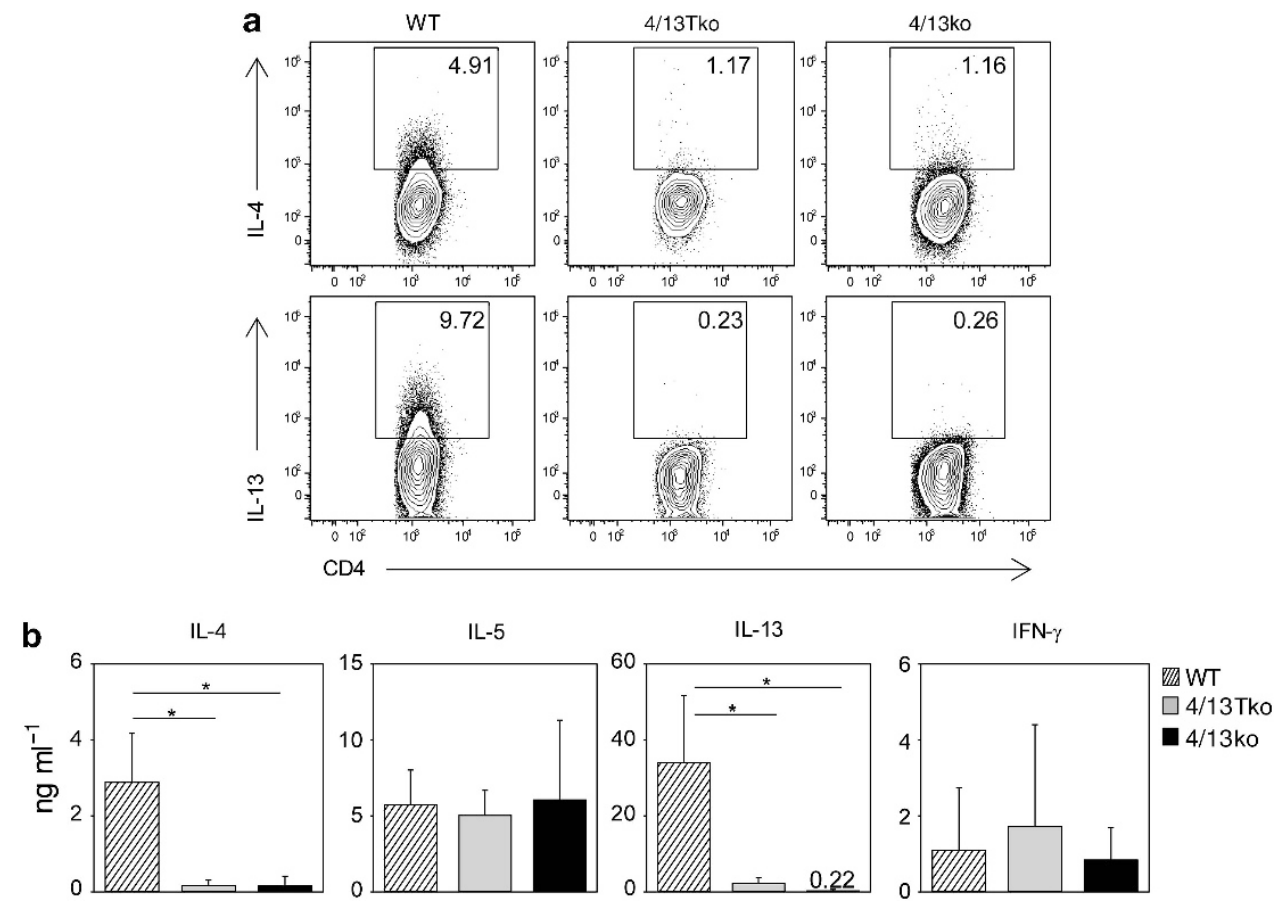

Figure 1 Cytokine production of T cells from wild-type (WT), 4/13Tko, and 4/13ko mice after in vitro stimulation. Total spleen cells of WT (hatched), 4/ 13Tko (gray), and 4/13ko (black) mice were cultured for 5 days in anti-CD3/anti-CD28-coated plates under T helper 2 (Th2)-polarizing conditions. (a) Dot plots show intracellular stainings of interleukin (IL)-4 and IL-13 in restimulated CD4 T cells. Samples were gated on live singlet CD4 ${ }^{+}$cells and results are representative of three independent experiments. (b) Concentrations of indicated cytokines in the supernatant measured by ELISA. Bars show the mean + s.d. of T-cell cultures from three individual mice per group and are representative of three independent experiments. ${ }^{*} P<0.05$ by two-tailed Student's $t$-test. ELISA, enzyme-linked immunosorbent assay; IFN- $\gamma$, interferon- $\gamma$; 4/13ko, IL-4/IL-13-deficient mice; 4/13Tko, T cell-specific IL-4/IL-13deficient mice.

sufficient for worm expulsion after infection with $N$. brasiliensis, whereas both IL-4/IL-13 $3^{-1-}$ and $\mathrm{Rag}^{-1-}$ mice alone show high worm burdens 9 days after infection. ${ }^{9}$ The caveat of this experiment was that the adoptive transfer system reflects a rather artificial situation with nonphysiologic lymphocyte numbers and secondary effects that may influence the efficiency of worm expulsion. However, the results argued for a critical role of innate effector cells as source of IL-4/IL-13. In support of this concept, it has been demonstrated that transfer of IL-13-producing ILC2s is sufficient for worm expulsion in IL-4/IL-13-deficient mice during primary infection with $N$. brasiliensis. ${ }^{10}$ However, it remains to be determined whether selective deletion of IL-4/IL-13 in ILC2s ablates the protection.

Depending on the infection model, IL- 4 and IL-13 have both redundant and unique functions. Surprisingly, after infections with $N$. brasiliensis, IL-4 is sufficient but not necessary for protection. ${ }^{11}$ In contrast, IL-13-deficient mice are not able to expel the parasite, ${ }^{12}$ suggesting an important role for this cytokine in initiating effector functions that clear the parasite. However, it was also noted that IL-4 expression is reduced in IL13-deficient mice after in vitro differentiation of $\mathrm{T}$ cells, ${ }^{13}$ and similarly IL-4-deficient mice produce reduced amounts of IL13 after helminth infection. ${ }^{14}$

As $N$. brasiliensis larvae mature and produce eggs in the gut, the clearance of the parasite especially depends on an intact intestinal immune response. It was reported that the recruitment of alternatively activated macrophages (AAMs) leads to increased intestinal smooth muscle activity that contributes to worm expulsion. ${ }^{15}$ Helminth infections also induce the differentiation of IL- $4 \mathrm{R} \alpha$-expressing intestinal epithelial cells into hyperplastic goblet cells that then secrete mucus and other effector molecules like RELM $\beta{ }^{16}$ Expression of RELM $\beta$ is induced by IL- 4 and IL- 13 but is independent of B cells, T cells, and AAMs. Both deletion of the IL- $4 \mathrm{R} \alpha$ and deletion of the cytokines IL-4/IL-13 lead to decreased goblet cell hyperplasia, resulting in a defective worm expulsion. ${ }^{17}$ Taken together, these results indicate that effector functions like the alternative activation of macrophages and goblet cell hyperplasia are of specific importance in the immune response against $N$. brasiliensis. However, so far it is not known whether fully differentiated Th2 cells are required as producers of IL- 4 and IL13 for induction of these effector functions and for protection or whether innate immune cells are sufficient. In order to further clarify this issue, we examined the role of T cell-derived IL-4/IL13 in response to infections with $N$. brasiliensis. Therefore, the immune responses of WT, T cell-specific IL-4/IL-13-deficient mice $\left(\mathrm{CD} 4 \mathrm{Cre} \times \mathrm{IL}-4 / \mathrm{IL}-13^{\mathrm{F} / \mathrm{F}}\right.$; in short $\left.4 / 13 \mathrm{Tko}\right)$ and IL-4/IL13 -deficient mice (4/13ko) were compared. We show that $\mathrm{T}$ cellderived IL-4/IL-13 was required for differentiation of Th2 cells, recruitment of innate immune cells, and alternative activation of macrophages in the lung and the intestine. Nevertheless, 4/ 13Tko and WT mice cleared the parasites with comparable efficiency, indicating that $\mathrm{T}$ cell-derived IL-4/IL-13 was 
dispensable for worm expulsion. In fact, innate IL-4/IL-13 appeared to be required and sufficient to induce goblet cell hyperplasia and secretion of important effector molecules like Mucin5ac and RELM $\beta$. These findings demonstrate that innate IL-4/IL-13 acts directly on intestinal epithelial cells to mediate downstream effector functions that are required for worm expulsion.

\section{RESULTS}

Deletion of a conditional IL-4/IL-13 allele in T cells does not result in a Th1-biased response

As the cytokines IL-4 and IL-13 play a critical role for expulsion of the gastrointestinal helminth $N$. brasiliensis, we thought to dissect the relative contributions of $\mathrm{T}$ cells and non- $\mathrm{T}$ cells as source for these cytokines. Therefore, we used BALB/c WT mice, $\mathrm{T}$ cell-specific IL-4/IL-13-deficient mice (CD4Cre $\times$ IL$\left.4 / \mathrm{IL}-13^{\mathrm{F} / \mathrm{F}} ; 4 / 13 \mathrm{Tko}\right)$ and IL-4/IL-13-deficient mice (4/13ko). To determine the efficiency of Cre-mediated deletion of the floxed IL-4/IL-13 allele, we first cultured splenic T cells under Th2-polarizing conditions in vitro. Intracellular stainings and enzyme-linked immunosorbent assays (ELISAs) from supernatants of restimulated $\mathrm{T}$ cells revealed efficient deletion of IL-4 and IL-13 in 4/13Tko and 4/13ko mice (Figure 1a,b). In contrast, cells from all three strains of mice produced similar amounts of IL-5, indicating that expression of this Th2associated cytokine was not affected in T cells from 4/13Tko and 4/13ko mice. Moreover, low but comparable amounts of the Th1-related cytokine interferon- $\gamma($ IFN- $\gamma)$ was expressed in all three mouse strains (Figure 1b). As exogenous IL-4 was used to drive Th2 polarization in this in vitro setting, we could not exclude that IL-5 and IFN- $\gamma$ levels would be differently regulated during an immune response to $N$. brasiliensis.

To address this point, we infected WT, 4/13Tko, and 4/13ko mice with $500 \mathrm{~L} 3$ larvae of $N$. brasiliensis and analyzed T-cell polarization at day 9 after infection. Expression of IL-4 and IL13 was upregulated in T cells of WT mice in lungs, tracheal lymph nodes (tLNs) and mesenteric lymph nodes (mLNs), whereas $\mathrm{T}$ cells from 4/13Tko and 4/13ko mice did not produce these cytokines (Figure 2a,b). However, high levels of IL-5 were still produced by $\mathrm{T}$ cells from all three groups of mice (Figure 2b). Furthermore, the lack of IL-4/IL-13-producing cells did not lead to a shift toward Th1 polarization, illustrated by the fact that IFN- $\gamma$-producing $\mathrm{T}$ cells did not increase significantly in different organs of 4/13Tko and 4/13ko mice (Figure 2a,b). In summary, we found that the lack of $\mathrm{T}$ cellderived or total IL-4 and IL-13 does not impair IL-5 production and does not lead to enhanced Th1 immune responses after both in vitro stimulation and helminth infection.

\section{T cell-derived paracrine IL-4/IL-13 is important for upregulation of Gata3}

Next, we wanted to determine whether Th2 polarization is generally affected in T cells from $N$. brasiliensis-infected 4/ 13Tko and 4/13ko mice. Therefore, expression of the Th2associated master transcription factor Gata3 was assessed by flow cytometry. Indeed, T cells from 4/13Tko and 4/13ko mice showed decreased expression of Gata3 in lungs, tLNs, and mLNs as compared with WT mice (Figure 3a,b). Furthermore, the frequency and total number of Gata $3^{+} \mathrm{CD} 4 \mathrm{~T}$ cells was higher in the lungs but not the LNs of 4/13Tko as compared with 4/13ko mice, suggesting that innate IL-4/IL-13-expressing cells may to some extent induce Gata3 expression in the lung, supporting recent findings in a model of allergic lung inflammation. ${ }^{18}$ The reduced Gata3 expression could be confirmed by studies of in vitro stimulation under Th2polarizing conditions. Even with high amounts of exogenous IL-4, T cells from 4/13Tko and 4/13ko mice showed reduced expression of Gata3 (Figure 3c). Consequently, the next step was to investigate whether this $\mathrm{T}$ cell-derived $\mathrm{IL}-4 / \mathrm{IL}-13$ is required in an autocrine (cell intrinsic) or paracrine manner. For that reason, mixed bone marrow chimeras with cells from congenic WT (CD45.1 CD90.2) and 4/13ko (CD45.2 CD90.1) mice were generated in a 1:1 ratio. This setting allowed us to determine whether IL-4/IL-13-deficient T cells were still able to develop into Gata3 ${ }^{+}$Th2-like cells when the cytokines were provided by WT Th2 cells in the same environment. After infection with $N$. brasiliensis, polarization, proliferation, and activation of T cells from WT and 4/13ko mice were compared. In all analyzed organs, namely lungs, mLNs, tLNs, and spleens, the percentages of $\mathrm{Gata}^{+} \mathrm{T}$ cells were similar in WT and 4/13ko cells (Figure 3d). In addition, proliferation, which was monitored via 5-bromo- $2^{\prime}$-deoxyuridine (BrdU) incorporation, and activation, where activated $\mathrm{T}$ cells were gated as $\mathrm{CD} 11 \mathrm{a}^{+}$and $\mathrm{CD} 62 \mathrm{~L}^{\text {low }}$ cells, was similar between WT and 4/13ko cells (Figure 3d). Altogether, these studies revealed that $\mathrm{T}$ cell-derived IL-4/IL-13 promotes expression of the Th2 master transcription factor Gata3. Importantly, these cytokines do not have to be produced by each developing Th2 cell itself, but can also be provided by other $\mathrm{T}$ cells in the environment.

\section{T cell-derived IL-4/IL-13 promotes mobilization and recruitment of basophils, eosinophils, and ILC2s after $N$. brasiliensis infection}

IL-4 and IL-13 induce a variety of Stat6-regulated chemokines that mobilize and recruit innate type 2 effector cells that have been shown to play important roles in the infection model of N. brasiliensis. For example, ILC2s are critical for worm expulsion after primary infection, ${ }^{10}$ whereas eosinophils and basophils contribute to protection after secondary infection. ${ }^{19-}$ ${ }^{21}$ Kinetic analysis of effector cell mobilization and recruitment in N. brasiliensis-infected IL-4eGFP reporter mice revealed that ILC2s increase in the lung already 5 days after infection, whereas an increase of Th2 cells, eosinophils, and basophils was not observed at that early time point. At day 9 after infection, eosinophils were the most abundant IL-4-expressing effector cells in the lung followed by Th2 cells, basophils, and very few ILC2s (Supplementary Figure S1a and b online). Next, we addressed the question of whether innate immune cells are affected by the selective deletion of IL-4/IL-13 in T cells. The mobilization of basophils, eosinophils, and ILC2s to the blood and recruitment to the lung was assessed 9 days after infection of WT, 4/13Tko, and 4/13ko mice (Figure 4a and 
a
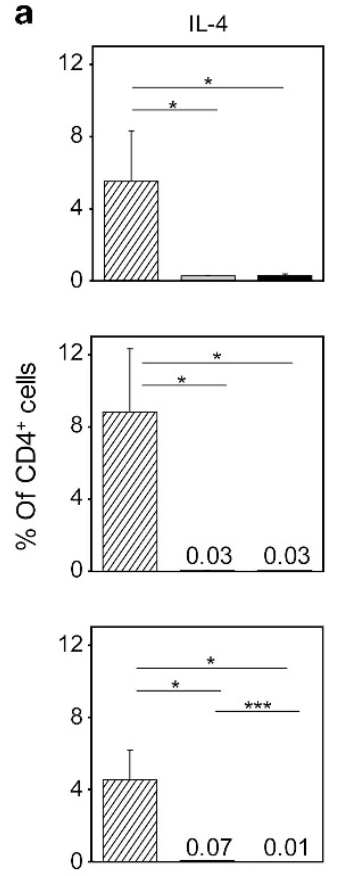

b
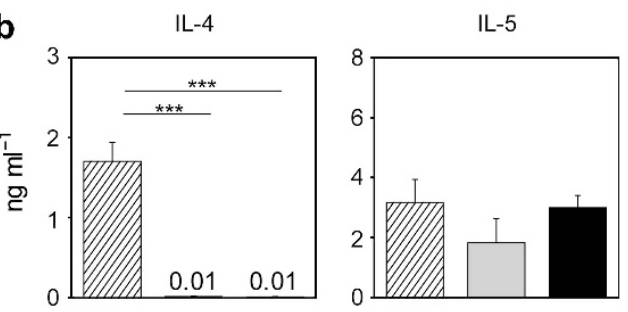
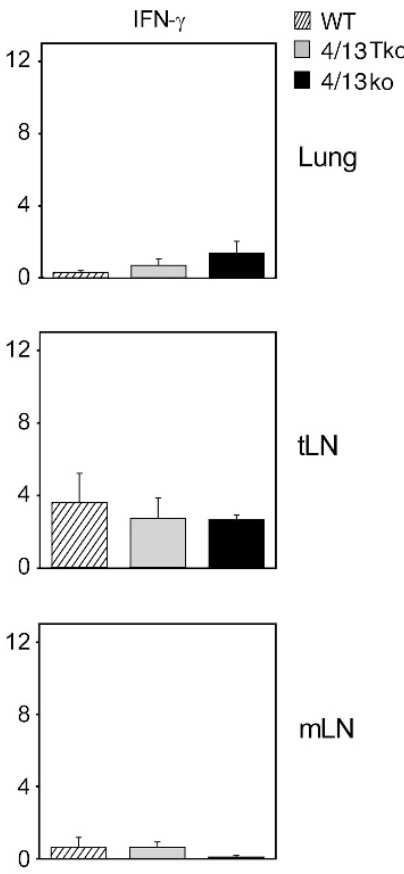
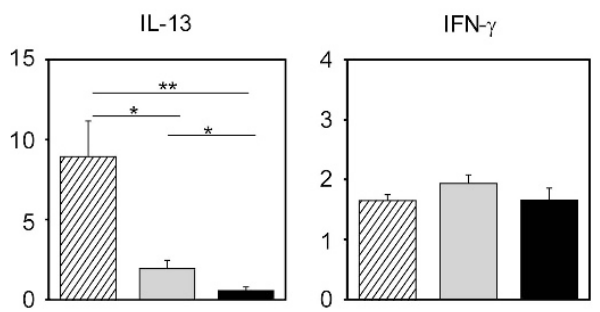

Figure 2 Cytokine production of T cells from WT, 4/13Tko, and 4/13ko mice after Nippostrongylus brasiliensis infection. WT (hatched), 4/13Tko (gray), and 4/13ko (black) mice were infected with $N$. brasiliensis and cytokine production was measured 9 days later after brief restimulation with phorbol-12myristate-13-acetate (PMA) and ionomycin. (a) Frequency of IL-4 $4^{+}$(left panels) and IFN- $\gamma^{+}$(right panels) cells among CD4 ${ }^{+} \mathrm{T}$ cells in the lung, tracheal lymph nodes (tLNs), and mesenteric lymph nodes ( $\mathrm{mLNs}$ ). Bars show the mean $+\mathrm{s}$.d. of three individual mice per group and are representative of three independent experiments. (b) Concentration of indicated cytokines in the supernatant measured by ELISA. Bars show the mean + s.e.m. of six individual mice per group from two independent experiments. ${ }^{\star} P<0.05$, ${ }^{* *} P<0.01$, ${ }^{* \star *} P<0.001$ by two-tailed Student's $t$-test. ELISA, enzyme-linked immunosorbent assay; IFN- $\gamma$, interferon- $\gamma$; IL, interleukin; 4/13ko, IL-4/IL-13-deficient mice; 4/13Tko, T cell-specific IL-4/IL-13-deficient mice; WT, wild type.

Supplementary Figure S2). The frequencies and total numbers of eosinophils and basophils were higher in the blood and lung of WT mice compared with 4/13Tko and 4/13ko mice (Figure $4 \mathbf{b}, \mathbf{c}$ ). ILC2s were mobilized to the blood with the same efficiency in all three strains of mice, but T cell-derived IL4/IL-13 promoted a relative increase of these cells in the lung (Figure $4 \mathbf{b}$ ). Interestingly, total ILC2 numbers were not reduced in the lungs of 4/13ko mice because of the increased numbers of leukocytes in the lungs of these mice (Figure 4c). In summary, these experiments indicate that both 4/13Tko and 4/13ko mice mobilize eosinophils and basophils less efficiently than WT mice after infection with $N$. brasiliensis and also the recruitment of effector cells to the lung is regulated by $\mathrm{T}$ cell-derived IL4/IL-13.

\section{T cell-derived IL-4/IL-13 is required for alternative activation of macrophages but not for worm expulsion}

So far, we found that a lack of T cell-derived IL-4/IL-13 impaired several effector mechanisms of type 2 immune responses such as Gata3 induction in $\mathrm{T}$ cells, the recruitment of innate effector cells to the lung, and IgE production (not shown). The next question was whether these impairments also affect the clearance of the parasite. Interestingly, we observed that worms were eliminated with comparable efficiency in WT and 4/13Tko mice, whereas 4/13ko mice still harbored more than 150 adult worms 9 days after the infection (Figure 5a). Because of this observation, we now sought to further analyze the role of $\mathrm{T}$ cell-derived IL-4/IL-13 for other effector mechanisms that have a protective effect during worm infections. Recently, several studies demonstrated that AAMs not only play an important role in the process of wound healing but also fulfill an immunomodulatory function in type 2 immune responses. For example, it could be shown that the elimination of AAMs blocked smooth muscle hypercontractility and impaired expulsion of $N$. brasiliensis. ${ }^{15}$ For this reason, we next investigated the accumulation of AAMs in the small intestine and the lung of infected WT, 4/13Tko, and 4/13ko mice. 
a
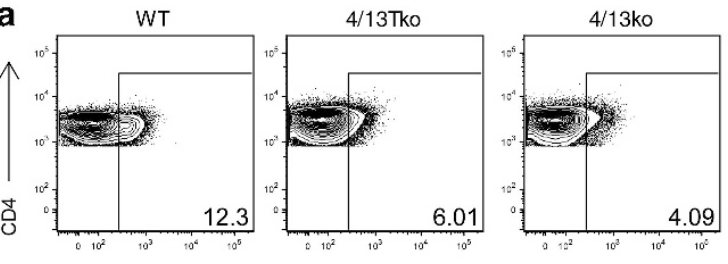

Gata3 $\longrightarrow$

b
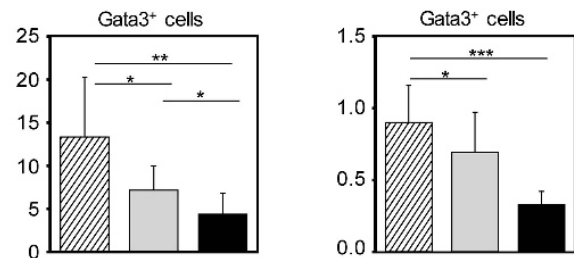

W WT

4/13Tko

- 4/13ko
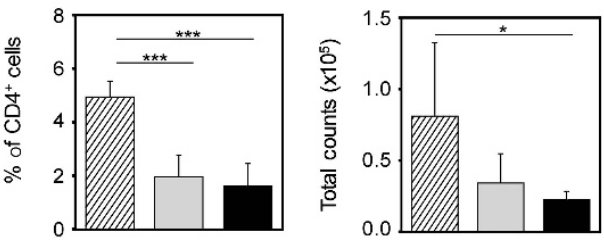

Lung
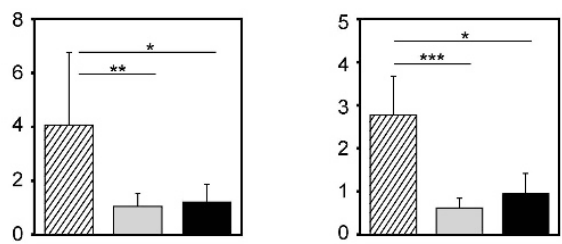

tLN
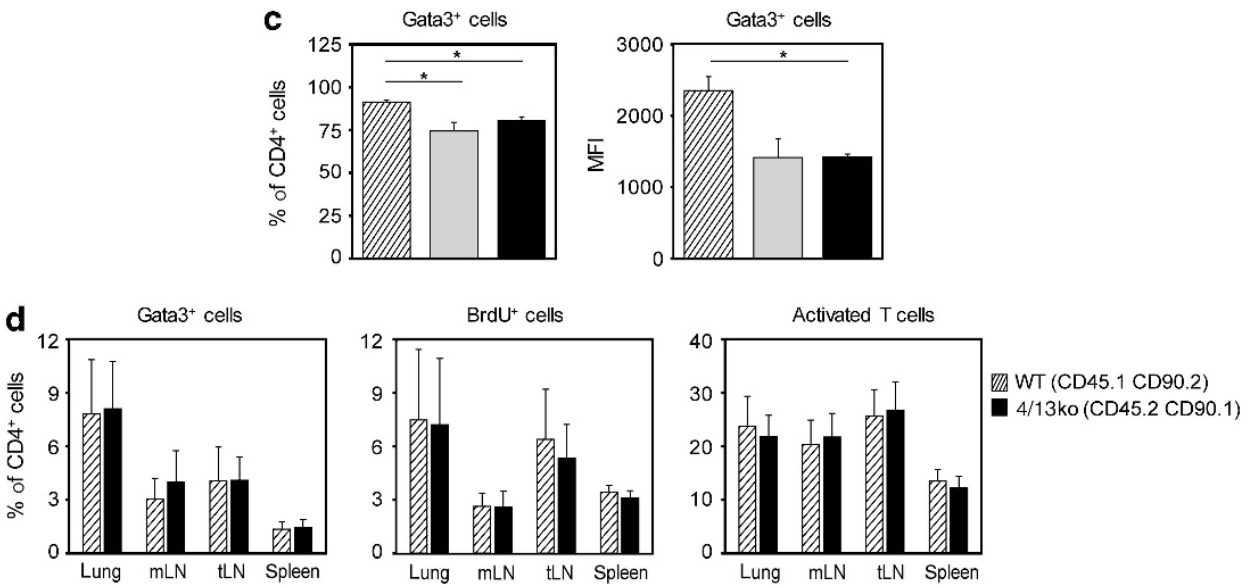

Figure 3 Analysis of Thelper 2 (Th2) cell polarization in the presence or absence of autocrine and paracrine IL-4 and IL-13. (a) Representative dot plots of $\mathrm{CD}^{+}{ }^{+} \mathrm{Gata}^{+}$cells of infected mice (gated on CD4 ${ }^{+}$cells). Gates were set accordingly to the corresponding isotype staining control. (b) Cells of indicated tissues from WT (hatched), 4/13Tko (gray), and 4/13ko (black) mice were stained for surface CD4 and intranuclear Gata3 9 days after Nippostrongylus brasiliensis infection. Graphs show percentages (left panels) and total numbers (right panels) of Gata3 ${ }^{+}$CD4 T cells. Bars show the mean + s.d. of three individual mice per group and are representative of three independent experiments. (c) Total spleen cells were cultured in the presence of anti-CD3/anti-CD28 antibodies for 5 days under Th2-polarizing conditions and stained for surface CD4 and intranuclear Gata3. Graphs show percentages (left panel) and mean fluorescence intensity (MFI, right panel) of Gata3 ${ }^{+}$CD4 T cells. Bars show the mean + s.d. of three individual experiments. (d) Mixed bone marrow chimeras that received cells from WT and 4/13ko mice were analyzed 9 days after infection with $N$. brasiliensis. Cells of indicated tissues were stained for surface CD4 and intranuclear Gata3 (left panel). T-cell proliferation was measured via flow cytometric analysis of BrdU incorporation (middle panel) and activated T cells were identified by gating on $\mathrm{CD}^{+}{ }^{+} \mathrm{CD} 11 \mathrm{a}^{+} \mathrm{CD} \mathrm{L}^{\text {low }}$ cells (right panel). Bars show the mean + s.d. of three to five individual mice. ${ }^{\star} P<0.05$, ${ }^{\star \star} P<0.01$, ${ }^{* * \star} P<0.001$ by two-tailed Student's $t$-test. IL, interleukin; $4 / 13 \mathrm{ko}$, IL-4/IL-13-deficient mice; $\mathrm{mLN}$, mesenteric lymph node; 4/13Tko, T cell-specific IL-4/IL-13-deficient mice; tLN, tracheal lymph node; WT, wild type.

RELM $\alpha$, also known as Fizz1, is a well-characterized marker for AAMs and was strongly upregulated in the small intestines of WT mice but not of 4/13Tko and 4/13ko mice
(Figure 5b). This result was also confirmed in the lung and the mLNs, where significantly more RELM $\alpha^{+}$AAMs could be detected in WT mice than in the other two mouse 
strains (Figure 5c). As it has been reported that intestinal epithelial cells and eosinophils can also express RELM $\alpha,{ }^{22,23}$ we included Ym1 and CD206, the mannose receptor, as other AAM markers in our studies. In immunofluorescence stainings of small intestine samples, $\mathrm{Ym} 1$ secretion and $\mathrm{CD} 206^{+} \mathrm{F} 4 / 80^{+}$ AAMs could only be detected in infected WT mice but not in 4/ 13Tko and 4/13ko mice (Figure 5d,e). Thus, we conclude that the recruitment of AAMs is dependent on T cell-derived IL-4/IL13 , but these cells were not required for worm expulsion.

\section{Innate IL-4/IL-13 induces goblet cell hyperplasia and secretion of effector molecules from intestinal epithelial cells}

As the differentiation of AAMs is not the critical mechanism for worm clearance, we next asked whether T cell-derived IL-4/IL13 was also required for goblet cell hyperplasia in the small intestine that is known to be an IL- $4 \mathrm{R} \alpha$-dependent process associated with efficient expulsion of $N$. brasiliensis. ${ }^{16}$ Interestingly, goblet cell hyperplasia was observed in both WT and 4/ 13Tko mice but not in 4/13ko mice, indicating that innate IL-4/ IL-13-expressing cells were sufficient for induction of goblet cell hyperplasia (Figure 6a). We further investigated whether Muc5ac and RELM $\beta$, two previously characterized antihelminthic effector molecules released from goblet cells and intestinal epithelial cells, were regulated by innate or adaptive IL-4/IL-13. Reverse transcriptase-PCR analysis of samples from the small intestines of $N$. brasiliensis-infected mice revealed that $M u c 5 a c$ and $R E L M \beta$ were upregulated in WT and 4/13Tko mice but not in 4/13ko mice (Figure 6b). Impaired RELM $\beta$ production in $4 / 13 \mathrm{ko}$ mice could also be confirmed at the protein level in the feces and by immunofluorescence staining (Figure $\mathbf{6 c}, \mathbf{d}$ ). Because of these results, we conclude a

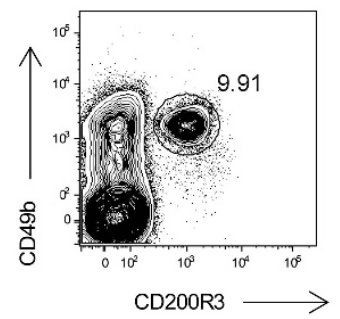

b
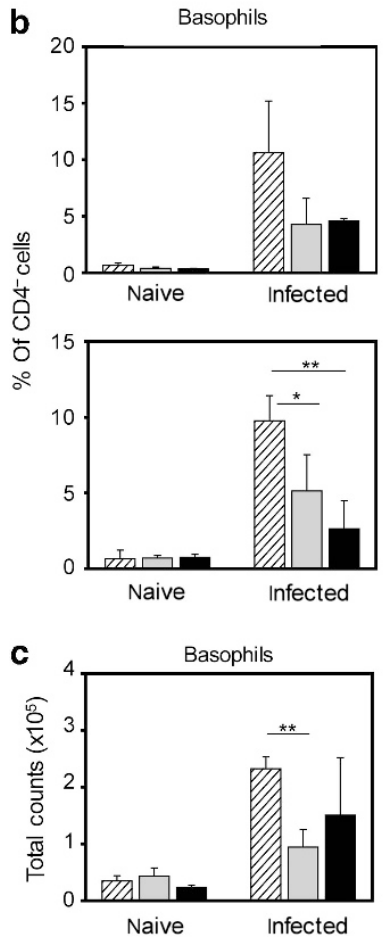
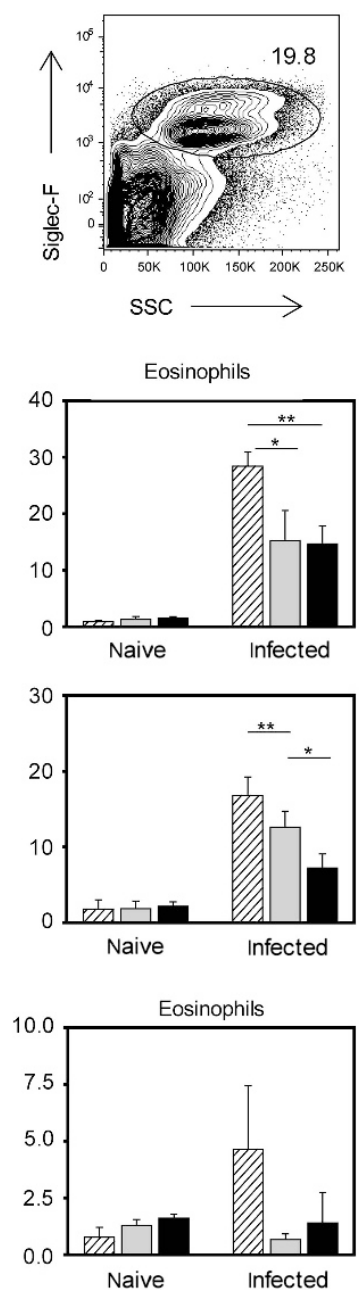
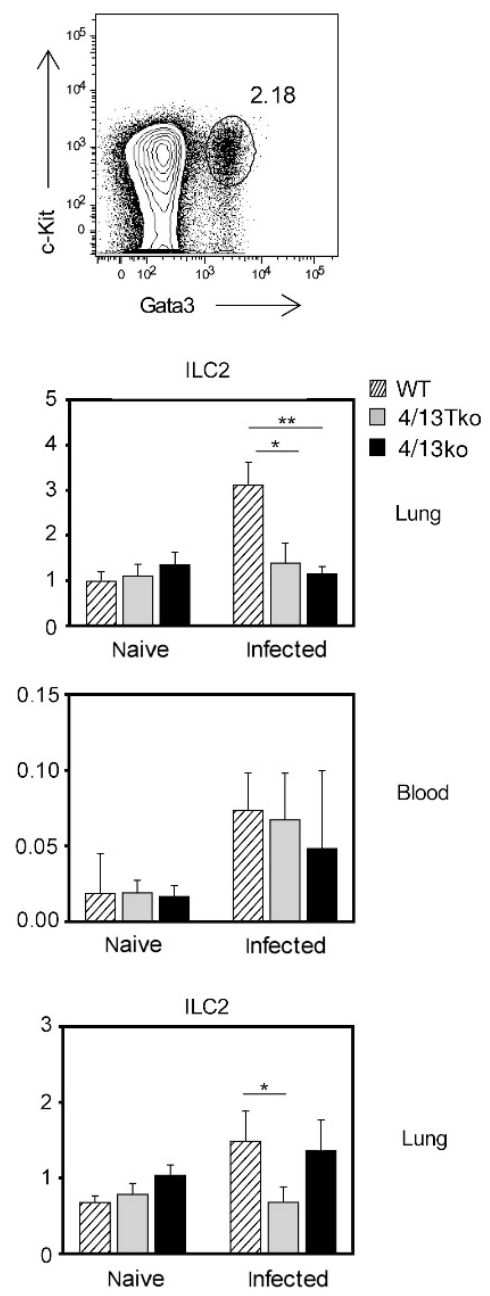

Figure 4 Nippostrongylus brasiliensis-induced mobilization and recruitment of basophils, eosinophils, and ILC2s in WT, 4/13Tko, and 4/13ko mice. Lung and blood cells from WT (hatched), 4/13Tko (gray), and 4/13ko (black) mice were analyzed by flow cytometry before (naive) and 9 days after (infected) $N$. brasiliensis infection. (a) Gating strategy for identification of CD4 ${ }^{-} \mathrm{CD} 49 \mathrm{~b}^{+} \mathrm{CD} 200 \mathrm{R} 3^{+}$basophils (left panel), $\mathrm{CD}^{-}{ }^{-}$Siglec- $\mathrm{F}^{+} \mathrm{SSC} \mathrm{Si}^{\text {hi }}$ eosinophils (middle panel), and $\mathrm{CD}^{-}{ }^{-} \mathrm{c}-\mathrm{Kit}^{+}{ }^{-} \mathrm{GATA} 3^{+}$ILC2s (right panel) shown for the lung of one infected WT mouse. Plots were gated on live singlet CD4 ${ }^{-}$cells. (b) Percentages and (c) total numbers of respective cell types. Bars show the mean $+\mathrm{s}$.d. of three individual mice per group and are representative of three independent experiments for the infected mice and one experiment for the naive mice. ${ }^{\star} P<0.05,{ }^{* \star} P<0.01,{ }^{* \star *} P<0.001$ by two-tailed Student's $t$-test. ILC2, innate type 2 lymphoid cell; 4/13ko, IL-4/IL-13-deficient mice; 4/13Tko, T cell-specific IL-4/IL-13-deficient mice; WT, wild type. 
a

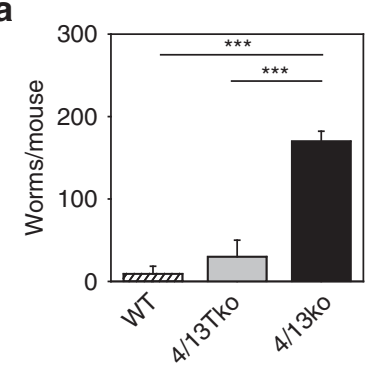

b

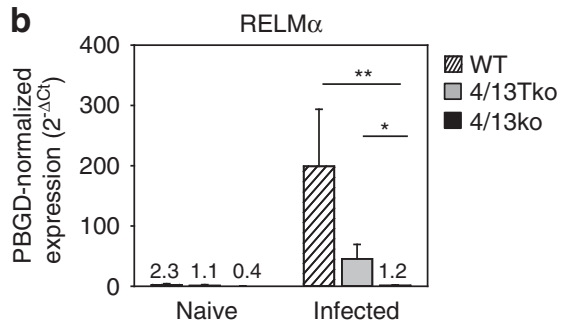

C
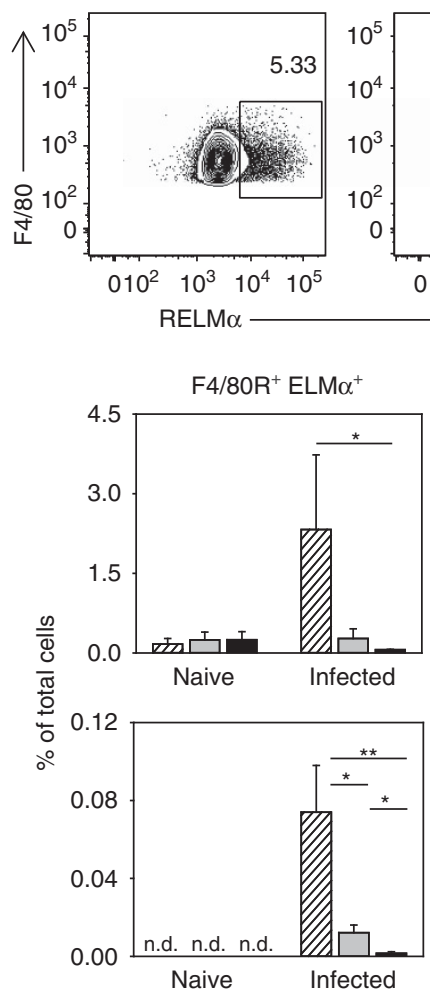

4/13Tko

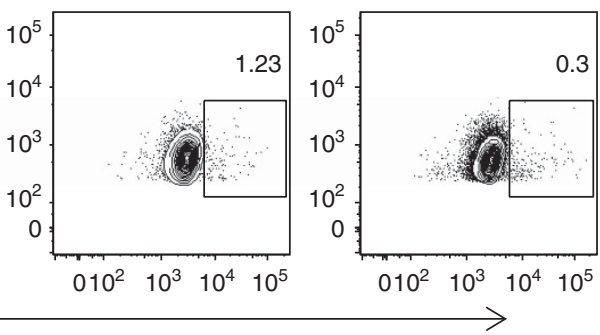

d
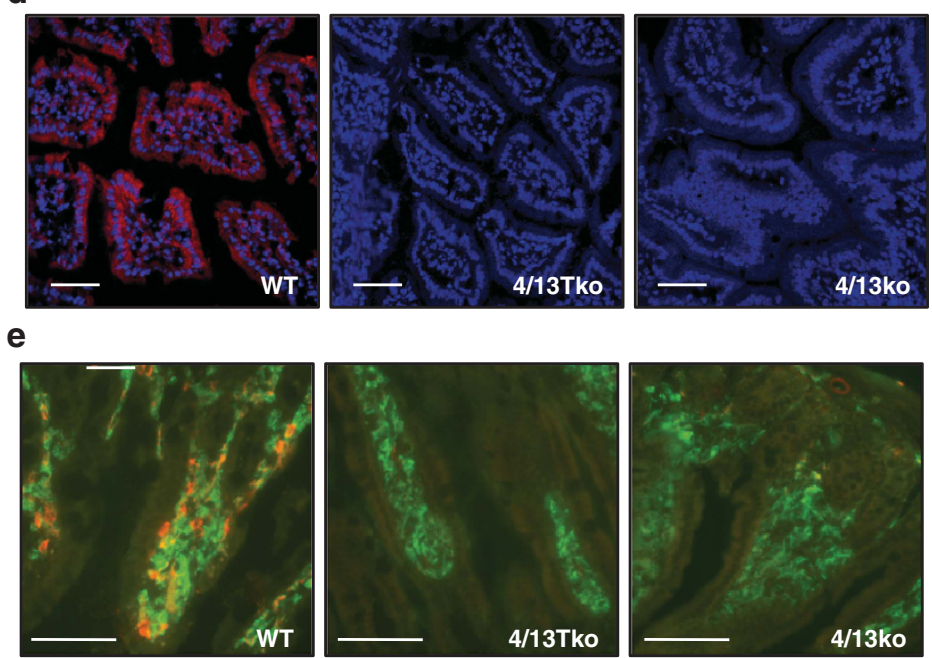
that induction of anti-helminthic effector molecules and goblet cell hyperplasia is largely dependent on IL-4/IL-13 from cells of the innate immune system and this finally leads to worm expulsion.

\section{DISCUSSION}

Infections with the gastrointestinal helminth $N$. brasiliensis constitute a suitable model for analyzing type 2 immune responses in mice. This study has concentrated on the role of $\mathrm{T}$
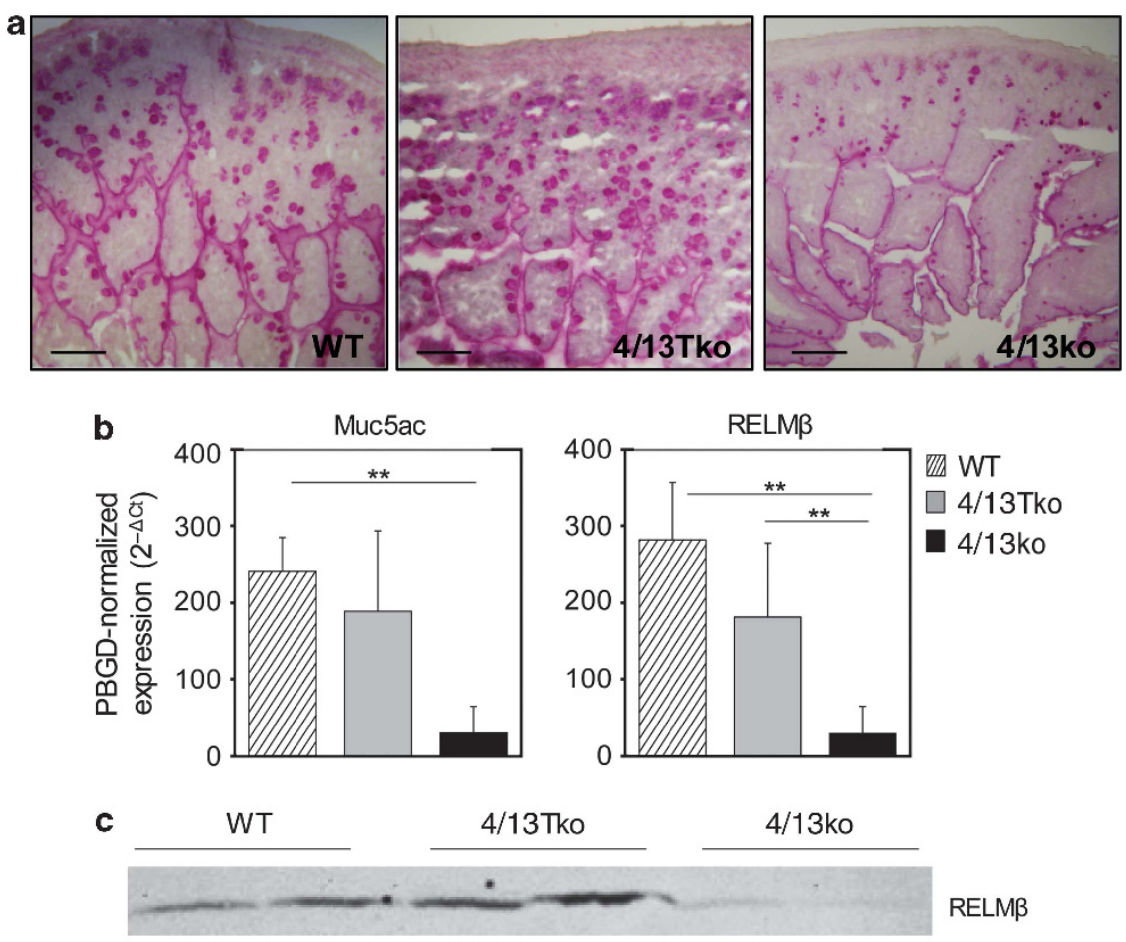

C
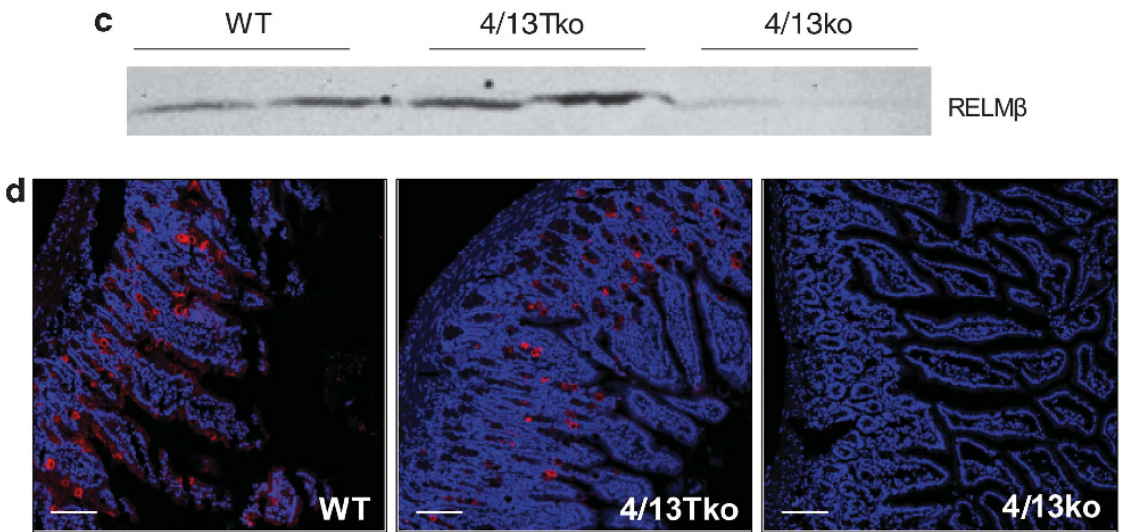

Figure 6 Activation of goblet cells in the small intestine of WT, 4/13Tko, and 4/13ko mice after Nippostrongylus brasiliensis infection. (a) Samples from the small intestines of infected mice were stained with periodic acid-Schiff. (b) Quantitative reverse transcriptase-PCR (RT-PCR) analysis for expression of Muc5ac (left panel) and RELM $\beta$ (right panel) from infected WT (hatched), 4/13Tko (gray), and 4/13ko (black) mice. Bars show the mean + s.d. of three individual mice per group and are representative of three independent experiments. ${ }^{* \star} P<0.01$ by two-tailed Student's $t$-test. (c) Western blot analysis for RELM $\beta$ protein in the feces of two infected mice per group. (d) Samples from the small intestines of infected mice stained with anti-RELM $\beta$ (red) and DAPI (blue). Scale bars $=100 \mu \mathrm{m}$. DAPI, 4,6 diamidino-2-phenylindole; 4/13ko, IL-4/IL-13-deficient mice; 4/13Tko, T cell-specific IL-4/IL-13-deficient mice; WT, wild type.

Figure 5 Worm expulsion and accumulation of alternatively activated macrophages (AAMs) in WT, 4/13Tko, and 4/13ko mice after Nippostrongylus brasiliensis infection. WT (hatched), 4/13Tko (gray), and 4/13ko (black) mice were analyzed for worm expulsion and expression of the AAM markers RELM $\alpha, Y m 1$, and CD206 before (naive) and 9 days after $N$. brasiliensis infection. (a) Bars show the number of adult $N$. brasiliensis worms in the small intestines of six to nine individual mice per group (mean + s.e.m.) 9 days after infection. (b) Quantitative reverse transcriptase-PCR (RT-PCR) analysis of small intestine tissue for expression of RELM $\alpha$. (c) Lung and mesenteric lymph node $(\mathrm{mLN})$ cells were stained for surface F4/80 and intracellular RELM $\alpha$. Top panels show representative dot plots of $F 4 / 80^{+}$RELM $\alpha^{+}$cells of infected WT mice (lung cells, gated on F4/80 ${ }^{+}$cells). Gates were set according to the corresponding isotype staining control. Graphs show percentages (left panels) and total numbers (right panels) of F4/80 ${ }^{+} \mathrm{RELM} \alpha^{+}$ AAMs in lung (middle panels) and $\mathrm{mLN}$ (bottom panels) samples. Bars show the mean $+\mathrm{s} . \mathrm{d}$. of three individual mice per group and are representative of three independent experiments for the infected mice and one experiment for the naive mice. ${ }^{*} P<0.05,{ }^{* *} P<0.01$, ${ }^{* * *} P<0.001$ by two-tailed Student's $t$ test. (d, e) Immunofluorescence staining of samples from the small intestine of infected mice stained with (d) anti-Ym1 (red) and DAPI (blue) or (e) anti-F4/ 80 (green) and anti-CD206 (red). Scale bars $=50 \mu \mathrm{m}$. DAPI, 4,6 diamidino-2-phenylindole; 4/13ko, IL-4/IL-13-deficient mice; 4/13Tko, T cell-specific IL4/IL-13-deficient mice; WT, wild type. 
cells and innate immune cells as producers of IL-4/IL-13 for the differentiation of Th2 cells and for the immune response against $N$. brasiliensis. We show that T cell-derived IL-4/IL-13 was required for recruitment of innate immune cells and alternative activation of macrophages in the lung and the intestine. Nevertheless, the lack of T cell-derived IL-4/IL-13 did not significantly impair the parasite clearance, whereas innate IL-4/IL-13 appeared to be required and sufficient to induce goblet cell hyperplasia and secretion of important effector molecules like Mucin5ac and RELM $\beta$, leading to worm expulsion.

We observed reduced mobilization of eosinophils and basophils to the blood and furthermore decreased recruitment of eosinophils, basophils, and ILC2s to the lung of 4/13Tko mice. But despite this impaired recruitment, 4/13Tko mice are still able to expel the parasite, indicating that, although in lower frequencies, innate immune cells are capable of initiating effective immune responses. Future experiments will have to further decipher the specific contribution of the different immune cells as multiple studies showed the contribution of different innate cell types in type 2 immune responses. One study showed that eosinophil-deficient $\Delta$ dblGATA mice or IL5 -deficient mice have higher worm burdens after primary and secondary infection with $N$. brasiliensis, ${ }^{24}$ although we previously found normal worm expulsion during primary infection of $\Delta$ dblGATA mice and impaired expulsion during secondary infection only when CD4 T cells had been depleted. ${ }^{9}$ We also reported previously that basophils are dispensable for worm expulsion during primary infection but contribute to protective immunity during secondary infections. ${ }^{20,25,26}$ In contrast, mast cell-deficient $\mathrm{c}-\mathrm{Kit}^{\mathrm{sash}}$ mice showed delayed worm expulsion during primary $N$. brasiliensis infection. ${ }^{25}$ At present, it remains unclear whether expression of IL-4/IL-13 in eosinophils and/or basophils contributes to the protective effects during secondary infection. ILC2s are potent producers of IL-13 and adoptive transfer experiments with ex vivo expanded ILC2s demonstrated that they are sufficient for expulsion of $N$. brasiliensis. ${ }^{10}$ However, experiments with selective deletion of IL-4/IL-13 in ILC2s have not been reported so far. Therefore, future experiments should specifically address the role of IL-4/IL-13 from eosinophils, basophils, mast cells, and ILC2s for worm expulsion during primary and secondary $N$. brasiliensis infection to reveal unique and redundant functions of these innate cell types for protective immunity.

Concerning the role of AAMs in N. brasiliensis infections, we observed that $\mathrm{T}$ cell-derived IL-4/IL-13 promotes AAM accumulation in lung and intestine. Surprisingly, the decreased recruitment of AAMs in 4/13Tko mice had no effect on the worm expulsion. In contrast to these findings, a previous study showed that a blockade of AAMs by clodronate-containing liposomes led to a blockade of smooth muscle hypercontractility and impaired parasite clearance. ${ }^{15}$ This discrepancy could be because of the different approaches as the effects on the recruitment and function of other cells by the liposome treatment was not analyzed. The results described here emphasize the role of intestinal epithelial cells and goblet cells for worm expulsion. These data are consistent with an earlier study where it was shown that IL-4/IL-13-induced differentiation of intestinal epithelial cells into hyperplastic goblet cells and their secretion of effector molecules was required for protection against gastrointestinal helminth infection. ${ }^{16}$ Our findings now show in a physiologic setting with conditional knockout mice that cells of the innate immune system rather than T cells are the essential IL-4/IL-13 producers for initiating these effector mechanisms.

In addition to the critical role of innate IL-4/IL-13 for worm expulsion, we observed that 4/13Tko mice show decreased expression of Gata3, the key transcription factor for Th2 cells, suggesting that $\mathrm{T}$ cell-derived IL-4/IL-13 promotes the development of Th2 cells. Interestingly, the experiments with mixed bone marrow chimeras revealed that the differentiation, activation, and proliferation of Th2 cells did not require these cytokines cell intrinsically but that they could be provided by other $\mathrm{T}$ cells in the environment. As an earlier study showed that autocrine IL-4 is also sufficient for the development of Th2 cells after adoptive transfer ${ }^{27}$ it can be concluded that $\mathrm{T}$ cell-derived IL-4 is required for Th2 differentiation but it can be provided either in an autocrine or paracrine manner.

In conclusion, we show that $\mathrm{T}$ cell-derived IL-4/IL-13 promoted Th2 polarization in a paracrine manner, as well as AAM differentiation and tissue recruitment of innate effector cells, whereas IL-4/IL-13 from cells of the innate immune system was required and sufficient for expulsion of $N$. brasiliensis. Future studies should therefore investigate how eosinophils, basophils, and ILC2s get activated and to what extent IL-4/IL-13 from each individual cell type contributes to worm expulsion during primary and secondary infection. Eosinophils and basophils express activating Fc receptors, suggesting that helminth-specific antibodies could mediate the activation of these cells. This would have implications for the design of effective vaccines that should favor the generation of long-lived plasma cells rather than memory Th2 cells.

\section{METHODS}

Mice. BALB/c mice were originally obtained from Charles River (Sulzfeld, Germany). IL-4/IL-13-deficient mice (4/13ko) ${ }^{28}$ were kindly provided by AN McKenzie (MRC Laboratory of Molecular Biology, Cambridge, UK). Mice with a loxP-flanked IL-4/IL-13 allele ${ }^{26}$ were crossed to CD4Cre mice ${ }^{29}$ to generate mice with selective deletion of both cytokines in T cells (4/13Tko mice) and backcrossed for more than 10 generations to $\mathrm{BALB} / \mathrm{c}$ background. IL-4/eGFP reporter mice (4get mice) $^{30}$ were kindly provided by RM Locksley (Howard Hughes Medical Institute, UC San Francisco, San Francisco, CA). All mice were housed according to institutional guidelines and used between 6 and 12 weeks of age. The animal experiments were approved by the Regierung von Mittelfranken and performed in accordance with the German animal protection law and the EU guidelines 86/809.

$\boldsymbol{N}$. brasiliensis infections and determination of worm burden. $N$. brasiliensis third-stage larvae (L3) were recovered from the cultured feces of infected rats and washed extensively in sterile $0.9 \%$ saline. Then, 500 larvae were injected into mice subcutaneously at the base of the tail. Mice were provided antibiotics-containing water $\left(2 \mathrm{gl}^{-1}\right.$ neomycin sulfate, $100 \mathrm{mgl}^{-1}$ polymyxin B; Sigma-Aldrich, St Louis, $\mathrm{MO}$ ) for the first 5 days after infection. Mice were analyzed at indicated 
time points after infection. Adult worms were counted in the small intestine after longitudinal dissection in RPMI-1640 medium (PanBiotech, Aidenbach, Germany) and incubation for $2 \mathrm{~h}$ at $37^{\circ} \mathrm{C}$.

In vitro culture of splenocytes. In vitro cultures were set up with total spleen cells at a density of $2 \times 10^{6}$ cells per well in a 24 -well plate that had been coated before with $0.5 \mu \mathrm{g} \mathrm{ml}{ }^{-1}$ anti-CD3 and anti-CD28 antibodies (eBioscience, San Diego, CA). Cells were cultivated in RPMI-1640 (PanBiotech) supplemented with 10\% fetal calf serum (Invitrogen, Carlsbad, CA), $2 \mathrm{~mm}$ L-glutamine, $100 \mathrm{U} \mathrm{ml}^{-1}$ penicillin, $10 \mathrm{U} \mathrm{ml}^{-1}$ streptomycin (Biochrom, Berlin, Germany), and $0.05 \mathrm{~mm}$ $\beta$-Mercaptoethanol (Merck Chemicals, Darmstadt, Germany). To induce Th2 differentiation, cells were cultured with IL-2 $\left(20 \mathrm{ng} \mathrm{ml}^{-1}\right.$; ImmunoTools, Friesoythe, Germany), IL-4 (20 ng ml ${ }^{-1}$; R\&D Systems, Minneapolis, MN), and anti-IFN- $\gamma\left(20 \mu \mathrm{g} \mathrm{ml}^{-1}\right.$; XMG1.2) for 3 days. On the following 2 days, the cells received fresh medium without IL-4 and anti-IFN- $\gamma$. On day 5 , cells were analyzed via flow cytometry and supernatants via ELISA.

Flow cytometry. Lung samples were digested with Collagenase Type VIII $\left(10 \mu \mathrm{g} \mathrm{ml}^{-1}\right.$; Sigma-Aldrich) for $30 \mathrm{~min}$ at $37^{\circ} \mathrm{C}$. Afterwards, lungs, spleens, tLNs, and mLNs were mechanically disrupted in a $70 \mu \mathrm{m}$ nylon strainer (Becton Dickinson, Franklin Lakes, NJ) to obtain single-cell suspensions. Next, cells were washed with fluorescenceactivated cell sorting (FACS) buffer (phosphate-buffered saline (PBS), $2 \%$ fetal calf serum) and incubated with anti-CD16/CD32 blocking antibody (clone 2.4G2; BioXcell, West Lebanon, $\mathrm{NH}$ ) for $5 \mathrm{~min}$ at room temperature followed by staining with antibody mixtures at $4{ }^{\circ} \mathrm{C}$. The following antibodies were used for surface staining.

Fluorescein isothiocyanate (FITC)-labeled anti-CD4 (clone RM45), PerCP-Cy5.5-labeled anti-CD4 (clone RM4-5), APC-eFluor 780labeled anti-CD4 (clone RM4-5), biotinylated anti-CD11a (clone M17/ 4), phycoerythrin (PE)-Cy7-labeled anti-CD62L (clone MEL-14), PElabeled anti-CD45.1 (clone A20), PerCP-Cy5.5-labeled anti-CD45.2 (clone 104), allophycocyanin (APC)-labeled anti-CD90.1 (clone HIS51), PE-Cy5-labeled anti-CD90.1 (clone HIS51), PE-Cy7-labeled anti-c-Kit (clone 2B8), APC-labeled anti-F4/80 (clone BM8), APClabeled anti-Sca-1 (clone D7), and eFluor 450-labeled anti-KLRG1 (clone 2F1) were purchased from eBioscience. Alexa Fluor 647-labeled anti-CD45.1 (clone A20), PE-labeled anti-CD200R3 (clone Ba13), and Alexa Fluor 647-labeled anti-CD49b (clone $\mathrm{HM} \alpha 2$ ) were purchased from BioLegend (San Diego, CA). Brilliant Violet 421-labeled antiSiglec-F (clone E50-2440) and FITC-labeled anti-IgE (clone R35-72) were purchased from BD Biosciences (Heidelberg, Germany). FITClabeled anti-CD90.1 (clone HIS51), APC-labeled anti-ICOS (clone 7E.17G9), and FITC-labeled anti-CD45.2 (clone A20) were purchased from Miltenyi Biotec (Bergisch-Gladbach, Germany). To detect biotinylated antibodies, V500 (BD Biosciences)- and PE (Southern Biotech, Birmingham, AL)-labeled streptavidin was used.

PE-labeled anti-GATA3 (clone TWAJ; eBioscience) and biotinylated anti-RELM $\alpha$ (PeproTech, Hamburg, Germany) were used for intracellular staining after cells had been fixed and permeabilized with the Foxp3/Transcription Factor Staining Buffer Set (eBioscience) according to the manufacturer's instructions.

For intracellular staining of cytokines, cells had been stimulated for $5 \mathrm{~h}$ with $40 \mathrm{ng} \mathrm{ml}^{-1}$ phorbol-12-myristate-13-acetate (SigmaAldrich) and $1 \mu \mathrm{g} \mathrm{ml}^{-1}$ ionomycin (Sigma-Aldrich). Brefeldin A $\left(5 \mu \mathrm{g} \mathrm{ml}^{-1}\right.$; Sigma-Aldrich) was added for the last $3 \mathrm{~h}$ of stimulation. After the cells were fixed in $4 \%$ paraformaldehyde (Roth, Karlsruhe, Germany), the following antibodies were used: PE-labeled anti-IL-4 (clone 11B11), APC-labeled anti-IL-4 (clone 11B11), Alexa Fluor 647labeled anti-IL-13 (clone eBio13A; all from eBioscience), and FITClabeled anti-IFN- $\gamma$ (clone XMG1.2; BioLegend).

Dead cells were excluded by staining with 4,6 diamidino-2phenylindole (Sigma-Aldrich) or fixable viability dye Alexa eFluor 780 (eBioscience). Samples were acquired on FACSCanto II (BD Biosciences) or MACSQuant Analyzer (Miltenyi Biotec). Doublets were excluded by electronic gating. Data were analyzed with FlowJo software (Tree Star, Ashland, OR).

Restimulations of $\mathrm{mLN}$ cells and ELISAs. $1 \times 10^{6}$ cells per well were plated on a 24-well plate and restimulated with phorbol-12-myristate13 -acetate $\left(40 \mathrm{ng} \mathrm{ml}^{-1}\right)$ and ionomycin $\left(1 \mu \mathrm{g} \mathrm{ml}^{-1}\right)$. After $24 \mathrm{~h}$, IL-4, IL-5, IL-13, and IFN- $\gamma$ concentrations in the supernatant were detected by standard ELISA. IL- 4 was measured with the anti-IL-4 monoclonal antibodies 11B11 for coating and BVD6-24G2 (BioLegend) for detection. IL-5, IL-13, and IFN- $\gamma$ were measured with ELISA kits from BD Biosciences and PeproTech.

Generation of mixed bone marrow chimeras. Bone marrow cells were prepared from the tibia and femur of donor mice and resuspended in PBS. Then, $2 \times 10^{6}$ mixed bone marrow cells from BALB/c CD90.2 CD45.1 and 4/13ko CD90.1 CD45.2 mice (1:1 ratio) were transferred into BALB/c_CD90.2_CD45.2 recipient mice that had been lethally irradiated with one dose of $600 \mathrm{rad}$ and 1 dose of $500 \mathrm{rad}$ given $5 \mathrm{~h}$ apart. Mice were treated for 8 weeks with antibiotics in the drinking water $\left(2 \mathrm{gl}^{-1}\right.$ neomycin sulfate, $100 \mathrm{mgl}^{-1}$ polymyxin $\mathrm{B}$; Sigma-Aldrich).

BrdU staining. On day 7 after N. brasiliensis infection, $36 \mathrm{~h}$ before analysis, mice were injected with $1 \mathrm{mg}$ BrdU (Sigma-Aldrich) in $100 \mu \mathrm{l}$ PBS intraperitoneally. The BrdU injection was repeated 24 and $12 \mathrm{~h}$ before analysis. Single-cell suspensions from mLNs, tLNs, lungs, and spleens were stained for surface CD4, CD45.1, CD45.2, and CD90.1, washed with PBS, and subjected to BrdU staining as previously described. $^{26}$

Quantitative reverse transcriptase-PCR. RNA was extracted from small intestine samples with the RNeasy Mini Kit (Qiagen, Hilden, Germany). Then, $1 \mu \mathrm{g}$ total RNA was used for first-strand complementary DNA synthesis using SuperScript III Reverse Transcriptase (Invitrogen-Life Technologies, Carlsbad, CA) and oligo(dT) primers. PCR was performed on the 7900HT Fast Real-Time PCR system (Applied Biosystems-Life Technologies, Carlsbad, CA) with fivefold diluted complementary DNA templates using SYBR Green I (Qiagen) and the following primer sequences: Porphobilinogen deaminase (PBGD) forward 5' -TGGTTGTTCACTCCCTGAAGG-3', PBGD reverse $5^{\prime}$-AAAGACAACAGCATCACAAGGGT-3', RELM $\alpha$ forward $5^{\prime}$-CCATAGAGAGATTATCGTGGA-3', RELM $\alpha$ reverse $5^{\prime}$ TGGTCGAGTCAACGAGTAAG-3', Muc5ac forward $5^{\prime}$-CTGTGA CATTATCCCATAAGCCC-3', Muc5ac reverse 5'-AAGGGGTAT AGCTGGCCTGA- $3^{\prime}$, RELM $\beta$ forward $5^{\prime}$-AAGCCTACACTGTG TTTCCTTTT-3', RELM $\beta$ reverse $5^{\prime}$-GCTTCCTTGATCCTTT GATCCAC- $3^{\prime}$. PBGD served as housekeeping gene. PCR conditions were the following: $15 \mathrm{~min}$ initial denaturation at $95^{\circ} \mathrm{C}, 42$ cycles with $30 \mathrm{~s}$ of denaturation at $95^{\circ} \mathrm{C}, 45 \mathrm{~s}$ of annealing at 58 or $50^{\circ} \mathrm{C}$, and $45 \mathrm{~s}$ of elongation at $72{ }^{\circ} \mathrm{C}$.

Immunofluorescence and periodic acid-Schiff staining. Cryosections ( $6 \mu \mathrm{m}$ thick) of paraformaldehyde-fixed small intestine samples were blocked with TNB-buffer (TSA amplification kit, Perkin Elmer, Waltham, MA) supplemented with $1 \%$ rat serum, $1 \%$ mouse serum, and $40 \mu \mathrm{g} \mathrm{ml}^{-1} \mathrm{CD} 16 / 32$-blocking antibody. Slides were stained with rabbit anti-mouse Ym1 antibody (polyclonal, STEMCELL Technologies, Grenoble, France) followed by Cy3-labeled goat anti-rabbit IgG (Jackson ImmunoResearch Laboratories, West Grove, PA) or APC-labeled rat anti-mouse F4/80 antibody (clone BM8, eBioscience) and Alexa Fluor 488-labeled rat anti-mouse CD206 (clone MR5D3, AbD Serotec, Oxford, UK). For detection of RELM $\beta$-expressing cells, slides were pretreated with sodium citrate buffer $(10 \mathrm{~mm}$ sodium citrate, $0.05 \%$ Tween-20, $\mathrm{pH} 6.0$ ) for $10 \mathrm{~min}$ at $90^{\circ} \mathrm{C}$. Sections were then blocked with $2 \%$ bovine serum albumin-PBS $+40 \mu \mathrm{g} \mathrm{ml}^{-1}$ CD16/32-blocking antibody and stained with rabbit anti-murine RELM $\beta$ antibody (PeproTech) followed by Cy3-labeled goat antirabbit IgG. Nuclei were counterstained with 4,6 diamidino-2-phenylindole. Furthermore, fixed small intestine sections were examined 
via periodic acid-Schiff staining (Sigma-Aldrich) according to the manufacturer's instructions. Pictures were acquired on LSM 700, Axio Vert.A1 or Axiostar plus (all Carl Zeiss, Jena, Germany). Image analysis was performed using the LSM image browser.

Western blot analysis. Stool lysates were prepared from infected mice (9 days after infection) by homogenization in lysis buffer containing $50 \mathrm{~mm}$ Tris- $\mathrm{HCl}$ (pH 7.5, Roth), $100 \mathrm{~mm} \mathrm{NaCl}$ (Roth), $1 \mathrm{~mm}$ EDTA (Roth), 1\% Nonidet P-40 (Sigma-Aldrich), $20 \mu \mathrm{g} \mathrm{ml}^{-1}$ leupeptin (Sigma-Aldrich), $16 \mu \mathrm{g} / \mathrm{ml}$ aprotinin (Sigma-Aldrich), and $0.2 \mathrm{~mm}$ phenylmethylsulfonyl fluoride (Sigma-Aldrich). Protein concentrations were determined by bicinchoninic acid assay (Thermo Fisher Scientific, Waltham, MA). The samples were denaturated at $95^{\circ} \mathrm{C}$ for 10 min and separated on a $15 \%$ sodium dodecyl sulfate/polyacrylamide electrophoresis gel. After transfer to a Immun-Blot PVDF Membrane (Bio-Rad Laboratories, Hercules, CA), the membrane was blocked with $5 \%$ milk powder (Roth) in Tris-buffered saline Tween at room temperature for $1 \mathrm{~h}$. The primary antibody, anti-RELM $\beta\left(0.2 \mu \mathrm{g} \mathrm{ml}^{-1}\right.$, PeproTech), was incubated with the membrane in $0.5 \%$ milk in Trisbuffered saline Tween at $4{ }^{\circ} \mathrm{C}$ overnight. After washing, the membrane was incubated with Rabbit True Blot Anti-Rabbit IgG horseradish peroxidase (eBioscience) at room temperature for $1 \mathrm{~h}$ and developed using the ECL Plus Kit (Perkin Elmer) according to the manufacturer's instructions.

Statistical analysis. Statistics were calculated by Student's $t$-test using SigmaPlot software (Systat Software Inc., San Jose, CA).

SUPPLEMENTARY MATERIAL is linked to the online version of the paper at http://www.nature.com/mi

\section{ACKNOWLEDGMENTS}

We thank D. Döhler, A. Matthies, K. Castiglione, and L. Handl for technical assistance, L. Gundel and M. Kirsch for animal husbandry, C. Bogdan for continuous support, and R. Willebrand and M. Otte for helpful comments on this manuscript. This work was supported by the Deutsche Forschungsgemeinschaft (GRK1660).

\section{AUTHOR CONTRIBUTIONS}

K.O. performed most experiments, C.S. analyzed the kinetics of effector cell recruitment, and K.O. and D.V. planned experiments and wrote the manuscript.

\section{DISCLOSURE}

The authors declared no conflict of interest.

(c) 2015 Society for Mucosal Immunology

\section{REFERENCES}

1. Hotez, P.J. et al. Helminth infections: the great neglected tropical diseases. J. Clin. Invest. 118, 1311-1321 (2008).

2. Ogilvie, B.M. \& Hockley, D.J. Effects of immunity of Nippostrongylus brasiliensis adult worms: reversible and irreversible changes in infectivity, reproduction, and morphology. J. Parasitol. 54, 1073-1084 (1968).

3. Zheng, W. \& Flavell, R.A. The transcription factor GATA-3 is necessary and sufficient for Th2 cytokine gene expression in CD4 Tcells. Cell 89, 587-596 (1997).

4. Nelms, K., Keegan, A.D., Zamorano, J., Ryan, J.J. \& Paul, W.E. The IL-4 receptor: signaling mechanisms and biologic functions. Annu. Rev. Immunol. 17, 701-738 (1999).

5. LaPorte, S.L. et al. Molecular and structural basis of cytokine receptor pleiotropy in the interleukin-4/13 system. Cell 132, 259-272 (2008).

6. Urban, J.F. Jr. et al. IL-13, IL-4Ralpha, and Stat6 are required for the expulsion of the gastrointestinal nematode parasite Nippostrongylus brasiliensis. Immunity 8, 255-264 (1998).
7. Schmidt, S. et al. Nippostrongylus-induced intestinal hypercontractility requires IL-4 receptor alpha-responsiveness by Tcells in mice. PLoS One 7, e52211 (2012).

8. Katona, I.M., Urban, J.F. Jr \& Finkelman, F.D. The role of L3T4 + and Lyt$2+\mathrm{T}$ cells in the IgE response and immunity to Nippostrongylus brasiliensis. J. Immunol. 140, 3206-3211 (1988).

9. Voehringer, D., Reese, T.A., Huang, X., Shinkai, K. \& Locksley, R.M. Type 2 immunity is controlled by IL-4/L-13 expression in hematopoietic noneosinophil cells of the innate immune system. J. Exp. Med. 203, 14351446 (2006).

10. Neill, D.R. et al. Nuocytes represent a new innate effector leukocyte that mediates type-2 immunity. Nature 464, 1367-1370 (2010).

11. Lawrence, R.A., Gray, C.A., Osborne, J. \& Maizels, R.M. Nippostrongylus brasiliensis: cytokine responses and nematode expulsion in normal and IL4-deficient mice. Exp. Parasitol. 84, 65-73 (1996).

12. McKenzie, G.J., Bancroft, A., Grencis, R.K. \& McKenzie, A.N. A distinct role for interleukin-13 in Th2-cell-mediated immune responses. Curr. Biol. 8, 339-342 (1998).

13. McKenzie, G.J. et al. Impaired development of Th2 cells in IL-13-deficient mice. Immunity 9, 423-432 (1998).

14. Barner, M., Mohrs, M., Brombacher, F. \& Kopf, M. Differences between IL$4 \mathrm{R}$ alpha-deficient and IL-4-deficient mice reveal a role for $\mathrm{LL}-13$ in the regulation of Th2 responses. Curr. Biol. 8, 669-672 (1998).

15. Zhao, A. et al. Th2 cytokine-induced alterations in intestinal smooth muscle function depend on alternatively activated macrophages. Gastroenterology 135, 217-225 (2008).

16. Herbert, D.R. et al. Intestinal epithelial cell secretion of RELM-beta protects against gastrointestinal worm infection. J. Exp. Med. 206, 2947-2957 (2009).

17. Marillier, R.G. et al. IL-4/IL-13 independent goblet cell hyperplasia in experimental helminth infections. BMC Immunol. 9, 11 (2008).

18. Halim, T.Y. et al. Group 2 innate lymphoid cells are critical for the initiation of adaptive T helper 2 cell-mediated allergic lung inflammation. Immunity 40 , 425-435 (2014).

19. Ohnmacht, C., Pullner, A., van Rooijen, N. \& Voehringer, D. Analysis of eosinophil turnover in vivo reveals their active recruitment to and prolonged survival in the peritoneal cavity. J. Immunol. 179, 4766-4774 (2007).

20. Ohnmacht, C. et al. Basophils orchestrate chronic allergic dermatitis and protective immunity against helminths. Immunity 33, 364-374 (2010).

21. Voehringer, D. Type 2 immunity is controlled by IL-4/IL-13 expression in hematopoietic non-eosinophil cells of the innate immune system. J. Exp. Med. 203, 1435-1446 (2006).

22. Nair, M.G. et al. Alternatively activated macrophage-derived RELM-\{alpha\} is a negative regulator of type 2 inflammation in the lung. J. Exp. Med. 206, 937-952 (2009).

23. Holcomb, I.N. et al. FIZZ1, a novel cysteine-rich secreted protein associated with pulmonary inflammation, defines a new gene family. EMBO J. 19, 4046-4055 (2000).

24. Knott, M.L. et al. Impaired resistance in early secondary Nippostrongylus brasiliensis infections in mice with defective eosinophilopoeisis. Int. J. Parasitol. 37, 1367-1378 (2007).

25. Ohnmacht, C. \& Voehringer, D. Basophils protect against reinfection with hookworms independently of mast cells and memory Th2 cells. J. Immunol. 184, 344-350 (2010).

26. Ohnmacht, C. \& Voehringer, D. Basophil effector function and homeostasis during helminth infection. Blood 113, 2816-2825 (2009).

27. Liu, Z. et al. IL-2 and autocrine IL-4 drive the in vivo development of antigenspecific Th2 Tcells elicited by nematode parasites. J. Immunol. 174, 2242 2249 (2005).

28. McKenzie, G.J., Fallon, P.G., Emson, C.L., Grencis, R.K. \& McKenzie, A.N. Simultaneous disruption of interleukin (IL)-4 and IL-13 defines individual roles in Thelper cell type 2-mediated responses. J. Exp. Med. 189, 15651572 (1999).

29. Lee, P.P. et al. A critical role for Dnmt1 and DNA methylation in T cell development, function, and survival. Immunity 15, 763-774 (2001).

30. Mohrs, M., Shinkai, K., Mohrs, K. \& Locksley, R.M. Analysis of type 2 immunity in vivo with a bicistronic IL-4 reporter. Immunity $15,303-311$ (2001). 